\title{
Miller, Manjari Chatterjee (2021): Why Nations Rise. Narratives and the Path to Great Power
}

New York: Oxford University Press. 208 Seiten. \$27.95

\author{
Christian Tuschhoff $\mathbb{D}$
}

Angenommen: 22. Dezember 2021 / Online publiziert: 13. Januar 2022

(C) Der/die Autor(en) 2022

Der Aufstieg und Fall von (Groß-)Mächten erfreut sich seit Langem der Aufmerksamkeit in den Internationalen Beziehungen und der Geschichtsschreibung. Bekannt sind vor allem die Arbeiten von Paul Kennedy, die neueste Hegemonieforschung von Alexander Cooley und Daniel Nexon, die einschlägigen Arbeiten zur Erforschung von Imperien von Herfried Münkler, Charles Maier oder Victor Bulmer-Thomas sowie die Machtübergangstheorie (A. F. Organski, Jacek Kugler), die als Zweig des (Neo-)Realismus zu sehen ist und in die Sozialtheorie des Rationalismus eingeordnet werden kann. Vor wenigen Jahren veröffentlichten die Wirtschaftswissenschaftler Daron Acemoglu und James Robinson ihr aufsehenerregendes Buch ,Why Nations Fail". Darin zeigen sie, wie unterschiedliche ausbeutende oder wohlstandsmehrende Institutionen Erfolg oder Scheitern von Nationen befördern. In ihrer jüngsten Arbeit „The Narrow Corridor“ ergänzen die beiden Autoren ihre Argumentation, indem sie der Beziehung zwischen Staat und Gesellschaft eine ausschlaggebende Rolle für Erfolg oder Misserfolg von Staaten zumessen.

Manjari Chatterjee Millers Buch reiht sich in dieses interdisziplinäre Forschungsfeld ein und verleiht der Forschung eine neue konstruktivistische Richtung. Der Aufstieg von Mächten erfordere zwar die Ausweitung von wirtschaftlichen und militärischen Ressourcen, aber diese notwendigen Bedingungen seien nicht hinreichend. Denn Ressourcen allein erzeugten keineswegs politische Absichten, eine Großmacht zu werden und selbst internationale Beziehungen zu gestalten. Dazu seien vielmehr Ambitionen notwendig, die ihren Ausdruck in Narrativen von Eliten fänden. Erst wenn sich im Wettbewerb der Ideen Narrative von Großmachtambitionen durchsetzen, verfolgten Staaten Strategien machtpolitischen Aufstiegs. Sie globalisierten ihre Autorität und beeinflussten Perzeptionen über ihren Status. Wenn aber Ambitions-

Christian Tuschhoff $(\bowtie)$

Fachbereich Politik- und Sozialwissenschaften, Freie Universität Berlin, Berlin, Deutschland

E-Mail: christian.tuschhoff@fu-berlin.de 
narrative fehlten bzw. sich nicht durchsetzten, übten Staaten machtpolitische Zurückhaltung und strebten keinen Großmachtstatus an. Aufstiegsstrategien seien gerade in der ersten Phase maßgeblich durch Anpassungsleistungen gekennzeichnet. Aufstrebende Mächte fügten sich in die jeweils bestehende internationale Ordnung ein und befolgten die etablierten Normen, anstatt sie revisionistisch infrage zu stellen. Zusammengenommen geben aber Narrative den Ausschlag, welche politischen Absichten Staaten verfolgten, deren Stärke zunähme. Diese Analyse verfügt über einen großen Vorteil im Vergleich zur traditionellen Machtübergangstheorie. Sie kann zusätzlich erklären, wie und warum mächtiger werdende Großmächte manchmal, aber eben nicht immer, ihr Machtpotenzial ausspielen.

Miller führt den Nachweis für Ihren Ursache-Wirkungs-Zusammenhang von Aufstieg und Zurückhaltung von Mächten, indem sie einzelne Fälle untersucht und einem Vergleich unterzieht. Die USA nach dem Bürgerkrieg, Japan nach der MeijiRevolution und die Volksrepublik China heute erfüllten alle Bedingungen für aufstrebende Staaten. Dagegen verzichteten die Niederlande Ende des 19. Jahrhunderts, Japan während und nach dem Kalten Krieg sowie Indien in der Gegenwart auf Großmachtambitionen, weil deren dominante Elitennarrative mit der Verfechtung von Großmachtideen unvereinbar gewesen seien. Den Aufstieg verdankten die USA und Meiji-Japan der Tatsache, dass sie die internationale Norm des Kolonialismus übernahmen und internalisierten. Im Gegensatz dazu lehnten die Niederlande die allgemeinen Prinzipien des Kolonialismus ab und praktizierten statt des ausbeuterischen einen wohlwollenden Kolonialismus. Die VR China übernahm die internationale Norm des Multilateralismus, während Japan und Indien diese Norm ablehnten und stattdessen auf Unabhängigkeit und Bilateralismus setzten.

Millers Buch sollte vor allem bei der an internationalen Fragen interessierten Öffentlichkeit, in Denkfabriken oder bei Außenpolitikeliten auf Interesse stoßen. Denn der Verweis auf die ausschlaggebende Bedeutung von Narrativen balanciert das einseitige Starren auf den wirtschaftlichen und militärischen Machtzuwachs von China aus, das zur allgegenwärtig beobachtbaren Schaffung eines neuen Feindbildes führt. Implizit zeigt Miller einen Weg, wie man China anders als durch Gegenmachtbildung beeinflussen könnte. Narrative und Normen sind der entscheidende Ansatzpunkt.

Leser*innen aus dem akademischen Bereich werden diese Stärke ebenfalls zu schätzen wissen, aber dennoch ein paar Kritikpunkte geltend machen. So vermisst man den Anschluss an die bisherige Literatur zur Zufriedenheit von Staaten z. B. Holsti oder Lebow. Außerdem wirken manche Analysen zu holzschnittartig. Dazu gehören vor allem die internationalen Normen der jeweiligen Epoche. Kolonialismus und Multilateralismus sind nur grobe Beschreibungen vielschichtiger internationaler Normengebäude, die viel flexibler und dynamischer sind, als Miller glauben macht. Deshalb boten sich z. B. Meiji-Japan auch unterschiedliche Nachahmungsmodelle (Frankreich, Großbritannien oder Preußen), aus denen es auswählte, wie Miller hervorhebt. Dichotome Unterscheidungen von Aufstieg vs. Zurückhaltung oder Anpassung vs. Revision vereindeutlichen eine viel komplexere Welt und geben ihr eine klare Kontur. Der Preis dafür ist, dass Spektren mit vielfältigen Grautönen ausgeblendet werden. So vermisst man beispielsweise Hinweise auf die institutionalistische Literatur, in der gezeigt wird, wie Großmachtkämpfe in internationalen Organisationen ausgetragen werden, die aber weder als Anpassung noch als Revisio- 
nismus verstanden werden können. Ferner gibt es keinen Hinweis, wie und warum sich ein bestimmtes Narrativ innenpolitisch im Ideenwettbewerb durchsetzen kann. Der letzte Beweis, dass ein Narrativ gewonnen haben muss, liegt in der Folgerung, dass Staaten aufsteigen oder sich zurückhaltend verhalten. Damit nähert sich die Beweisführung aber sehr einer Tautologie, weil das Narrativ ja die ausschlaggebende Ursache für das unterschiedliche Großmachtverhalten darstellt. Und schließlich vermisst man Hinweise, wie und warum Mächte von einer anpassenden auf eine revisionistisch-transformatorische Strategie umsteigen.

Das Buch über den Aufstieg und die Zurückhaltung von Großmächten ist eine wichtige und notwendige Ergänzung der Literatur aus konstruktivistischer Perspektive. Die klare Gliederung und gut lesbare Sprache sind wesentliche Qualitätsmerkmale. Man wird aber dem eingeschlagenen neuen Weg der Forschung noch weiter und vertieft nachgehen müssen, bevor man vom politikwissenschaftlichen Nutzen der beschriebenen Ursache-Wirkungs-Beziehung vollständig überzeugt ist. Ein vielversprechender Weg dahin könnte sein, soziale Beziehungen als immaterielle Erfolgsfaktoren zu untersuchen, wie Hall und Lamont vorgeschlagen haben.

Funding Open Access funding enabled and organized by Projekt DEAL.

Open Access Dieser Artikel wird unter der Creative Commons Namensnennung 4.0 International Lizenz veröffentlicht, welche die Nutzung, Vervielfältigung, Bearbeitung, Verbreitung und Wiedergabe in jeglichem Medium und Format erlaubt, sofern Sie den/die ursprünglichen Autor(en) und die Quelle ordnungsgemäß nennen, einen Link zur Creative Commons Lizenz beifügen und angeben, ob Änderungen vorgenommen wurden.

Die in diesem Artikel enthaltenen Bilder und sonstiges Drittmaterial unterliegen ebenfalls der genannten Creative Commons Lizenz, sofern sich aus der Abbildungslegende nichts anderes ergibt. Sofern das betreffende Material nicht unter der genannten Creative Commons Lizenz steht und die betreffende Handlung nicht nach gesetzlichen Vorschriften erlaubt ist, ist für die oben aufgeführten Weiterverwendungen des Materials die Einwilligung des jeweiligen Rechteinhabers einzuholen.

Weitere Details zur Lizenz entnehmen Sie bitte der Lizenzinformation auf http://creativecommons.org/ licenses/by/4.0/deed.de. 